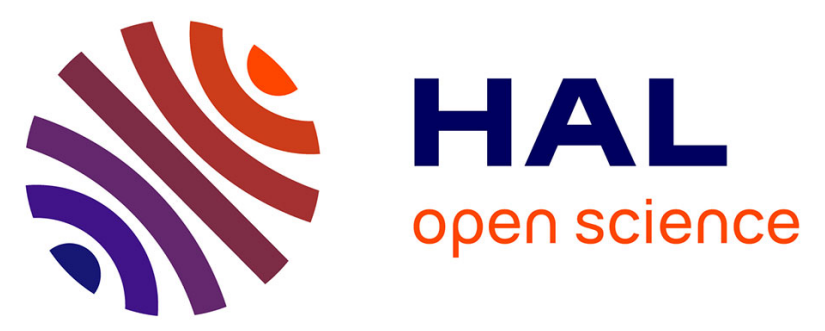

\title{
Assessment of ROC curves for inspection of random fields
}

Franck Schoefs, Alexandre Clément, Anthony Nouy

\section{To cite this version:}

Franck Schoefs, Alexandre Clément, Anthony Nouy. Assessment of ROC curves for inspection of random fields. Structural Safety, 2009, 31 (5), pp.409-419. 10.1016/j.strusafe.2009.01.004 . hal01005279

\section{HAL Id: hal-01005279 \\ https://hal.science/hal-01005279}

Submitted on 9 Aug 2017

HAL is a multi-disciplinary open access archive for the deposit and dissemination of scientific research documents, whether they are published or not. The documents may come from teaching and research institutions in France or abroad, or from public or private research centers.
L'archive ouverte pluridisciplinaire HAL, est destinée au dépôt et à la diffusion de documents scientifiques de niveau recherche, publiés ou non, émanant des établissements d'enseignement et de recherche français ou étrangers, des laboratoires publics ou privés. 


\title{
Assessment of ROC curves for inspection of random fields
}

\author{
F. Schoefs, A. Clément, A. Nouy \\ Research Institute in Civil Engineering and Mechanics (GeM), Nantes Atlantic University, Ecole Centrale Nantes, UMR CNRS 6183, 2 rue de la Houssinière, \\ B.P. 92208, 44322 Nantes Cedex 3, France
}

\begin{abstract}
Inspection by non-destructive testing (NDT) techniques of existing structures is not perfect and it has become a common practice to model their reliability in terms of probability of detection (PoD), probability of false alarms (PFA) and receiver operating characteristic (ROC) curves. These results are generally the main inputs needed by owners of structures in order to achieve inspection, maintenance and repair plans (IMR). The assessment of PoD and PFA is even deduced from intercalibration of NDT tools or from the modelling of the noise and the signal. In this last case when the noise and the signal depend on the location on the structure PoD and PFA are spatially dependent. This paper presents how to define PoD and PFA when damage and detection are stochastic fields or spatially dependent. Corrosion of coastal structures in harbours is considered for illustration and ROC curves are deduced. Identification of probability density functions on polynomial chaos is shown to be more suitable than predefined probability distribution functions (pdf) in view of fitting noise and signal plus noise distributions.
\end{abstract}

\author{
Keywords: \\ ROC curves \\ Probability of detection \\ Inspections \\ Stochastic fields \\ Corrosion \\ Polynomial Chaos
}

\section{Introduction}

The actual challenge of the maintenance of a set of structures (harbours, bridges, etc.) needs to find the optimum balance between the increasing number of deteriorating structures and the limited funds available for their upkeep [1-7]. The demolition and replacement of large engineering structures results in high economic and environmental costs, further increasing the need for efficient management plans to maintain these structures [8$10]$. Reassessment of existing structures needs to up-date materials properties. In a lot of cases, on site inspection are needed and in some cases visual inspection is not sufficient. For example nondestructive-techniques (NDT) tools are required for the inspection of coastal and marine structures where marine growth acts as a mask or underwater zone gives harsh condition of visual inspection. In these fields, the cost of inspection can be prohibitive and an accurate description of the performance on-site of NDT tools must be provided. During the last decade, the concepts of probability of detection (PoD), probability of false alarm (PFA) [11], probability of indication [12-14] have been proved to be suitable when performing risk-based-inspection [15-18] or management of networks [19]. They allow introducing the cost/benefit of NDT tools in a complete risk analysis. The paper focuses on their modelling in the case of inspection on structures. The probability of detection is closely linked to the level of the detection threshold and the size of the defect. When this size of defect is randomly spatially distributed, then, the probability of detection is spatially dependent. Knowing the distribution of the noise on measurements due to the decision-chain "physical measurement-decision on defect measurement-transfer of information" [20], the harsh environment of inspection and the complexity of the protocol (link diver-operator), the probability of false alarm can be defined. Then for a set of defects and given NDT tool and operator, couples (PoD;PFA) called receiver-operating-characteristics (ROC) can be assessed. Mainly in the case of very harsh conditions of inspection or of several diver experiences, ROC curves that link the probability of detection and the probability of false alarm are plotted. First we review in this paper the basic definitions of PoD, PFA and ROC. The way to introduce these concepts as decision aid tools is focused on. Then these definitions are extrapolated in the case of spatially dependent deterioration stochastic processes. Finally to illustrate these concepts, the paper focuses on generalized corrosion on steel-piles in coastal area. First, more than 1000 measurements of residual steel thickness are analyzed. They have been obtained from an ultrasonic NDTtool on a quay in order to analyze the probability distribution function that gives the best fit of defect distribution.

Moreover, a particular protocol during inspection allows defining and assessing the noise on measurements and discussing its spatial dependency. Two models of noise are suggested: the first one consists in considering one independent random variable by 
level of inspection and the second one consists in gathering data by area which leads to get one independent random variable by zone (tidal zone and underwater zone).

\section{Probabilistic modelling of inspection results based on detection theory}

\subsection{Theoretical background and basic concepts for PoD and PFA}

The most common concept which characterizes inspection tool performance is the probability of detection (PoD). Let $a_{d}$ be the minimal defect size, under which it is assumed that no detection is done. Parameter $a_{d}$ is called detection threshold in the following. Thus, the probability of detection is defined as:

$P o D=P\left(\widehat{d} \geqslant a_{d}\right)$

where $\hat{d}$ is the measured defect size. The detection threshold $a_{d}$ is a deterministic parameter or a random variable. In the case where $a_{d}$ is deterministic, this definition implies that PoD is a monotonic decreasing function of $a_{d}$. Detection theory gives the theoretical background for defining PFA, once given probability density functions $f_{\text {signal }}$ and $f_{\text {noise }}$, respectively of (signal+noise) and noise. Noise depends on environmental conditions, human interference and the nature of what is being measured. Then PoD and PFA have the following expressions (2) and (3):

$P o D=\int_{a_{d}}^{+\infty} f_{\text {signal }}(\widehat{d}) d \widehat{d}$

$P F A=\int_{a_{d}}^{+\infty} f_{\text {noise }}(\eta) d \eta$

Fig. 1 illustrates the probability density function and computation of PFA and PoD for a given detection threshold in the case where (signal+noise) and noise are normally distributed.

\subsection{Building of receiver operating characteristic curve}

For a given detection threshold the couple (PFA, PoD) allows defining N.D.T performance; this is the receiver operating characteristic (abbreviated R.O.C.). This couple can be considered as coordinates of a point in the plane (PFA, PoD). If we consider that $a_{d}$ takes values in the range ] $-\infty ;+\infty$ [, this point belongs to a curve called R.O.C curve. It is a parametric curve with parameter $a_{d}$ in Eqs. (2) and (3). R.O.C. curve plotted on Fig. 2 is computed with

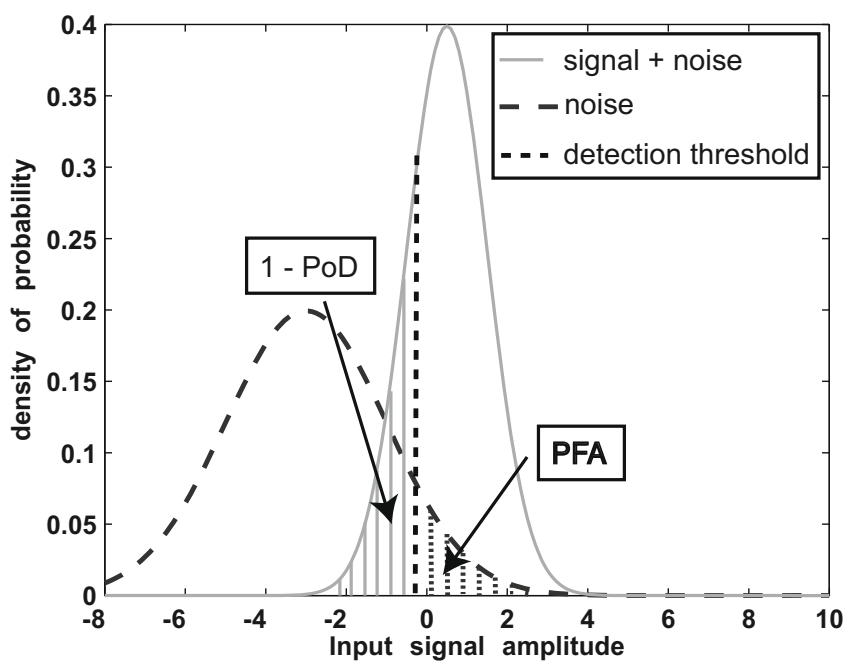

Fig. 1. Illustration of PoD and PFA (signal and noise normally distributed).

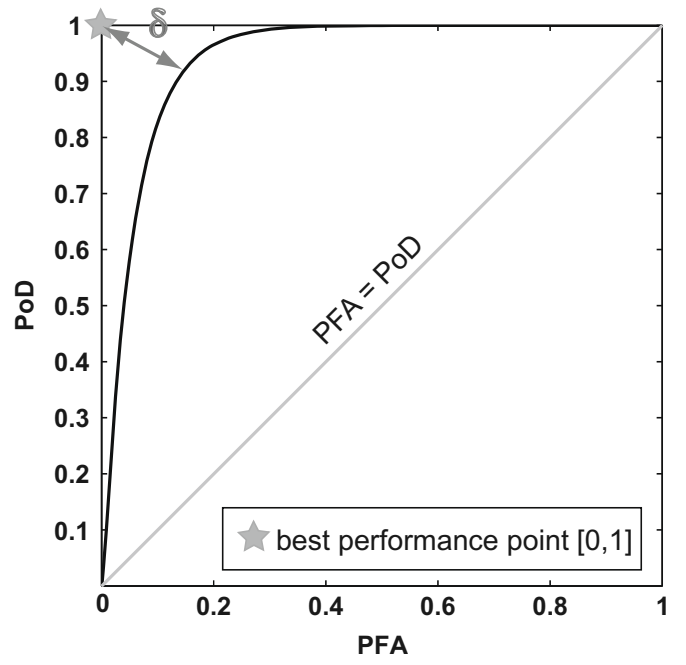

Fig. 2. Receiver operating characteristic curve.

the pdf presented on Fig. 1 corresponding to normal distributions. From a theoretical point of view, this is a curve corresponding to a monotonically increasing function, always lying above diagonal $(\mathrm{PFA}=\mathrm{PoD})$, and whose first derivative is closely linked to the sensitivity to the receiver $[21,22]$. The diagonal line running from lower left to upper right (curve "PFA=PoD") is the line of no "performance", since in that case the inspection result is the same, no matter what the observation is [13].

Looking for the best detection performances, the probability of detection should always take larger values than the probability of false alarm (low noise sensitivity). We have then: PoD $\geqslant$ PFA. When reading ROC curves, one must keep in mind that the probability of false alarm depends on the noise and detection threshold only. It does not depend on defect size except if the noise depends on defect size itself. The operator adjusts the device for example to detect smaller defects when the current adjustment does not give any signal. Probability of detection is a function of the detection threshold, the defect size, and the noise. Thus, for a given detection threshold, the probability of false alarm is a constant, but the probability of detection is an increasing function of the defect size. ROC curve is a fundamental characteristic of the NDT tool performance for a given defect size. Perfect tool is represented by a ROC curve reduced to a single point whose coordinates are: $(P F A, P o D)=$ $[0,1]$. The distance between this "best performance point" and the ROC curve is a measure of the NDT ability [11]. Different theoretical ROC curves, corresponding each one to different signal/ noise ratio of NDT tool are presented in [13].

When assessing a ROC curve, the challenge is then to get discrete values for PFA and PoD in given conditions [23,24] or to model the (signal+noise) and noise distributions on-site. We follow here the second approach. Note that the PFA is named PFI (Probability of False Indication) too. The definition of discrete values for PFI is generally expressed as a percentage of false indication on the inspected length $[11,25]$. Finally, as the number of samples is limited, authors provide generally confidence bounds: 90\% POD for example $[23,26]$.

\section{Modelling of inspection results for stochastic deterioration models}

\subsection{Spatial dependency of PoD and PFA}

In some cases, the performance of NDT tools depends on the location of the point to be inspected on the structure. As illustration, 


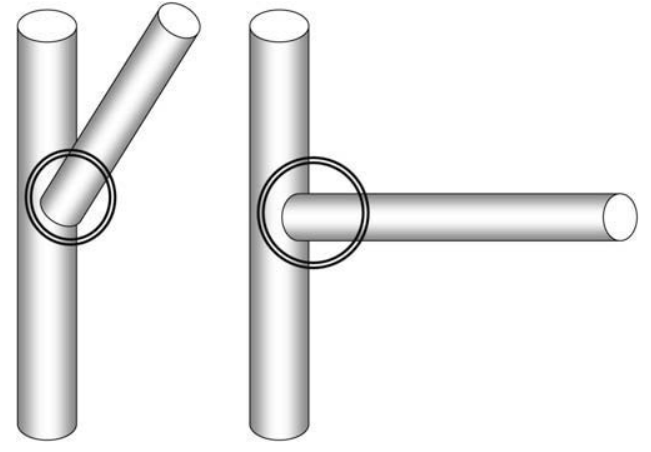

Fig. 3. Inspections on hot-spots on Y-joint (left) and T-joint (right).

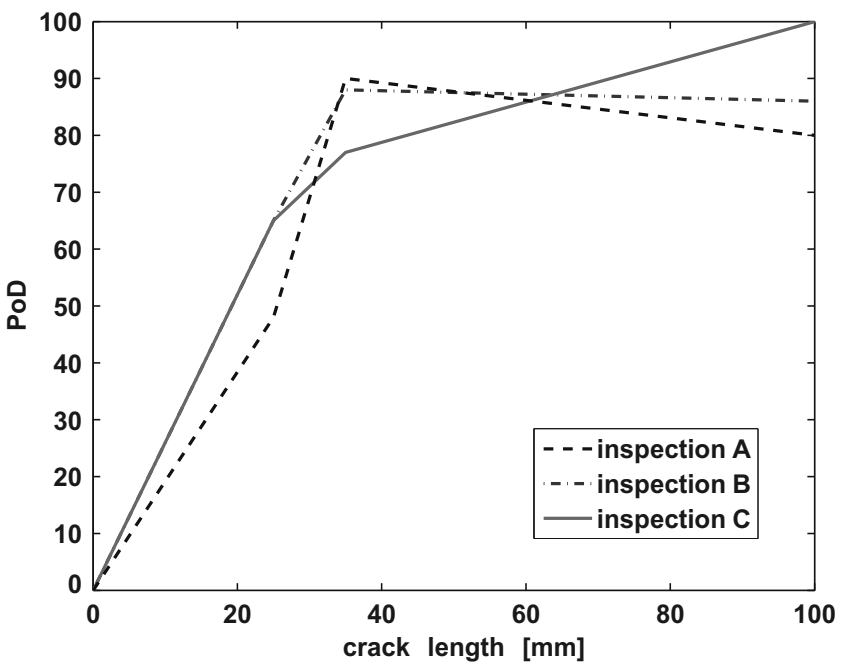

Fig. 4. PoD curves inspection of welded joints by 3 societies A, B and C.

let us consider the inspection of welded joints of offshore platforms with some techniques such as MPI (Magnetic Particle Inspection); the probability of detection of a given crack at the hot-spot of a Y-joint (inside the circle on Fig. 3 left) is lower than the probability of detection of the same crack at the hot-spot of a T-joint (inside the circle on Fig. 3 right). It has been observed during ICON project (InterCalibration of Offshore Ndt) $[23,27,28]$. When the number of samples is fair some PoD couldn't be monotonic decreasing functions due to statistical bias. Fig. 4 illustrates this case with the plot of three PoD evolutions with crack size obtained after inspections of the same samples by three inspection societies A, B and C during the ICON project. Inspectors from societies $B$ and $C$ encountered some difficulties with defects of length $100 \mathrm{~mm}$ because they were on Y-joints. When locations of defects are on welded joints, the corresponding PoD and PFA should be changed according to the access, the luminosity and the wave shaking for instance. When defects are continuous fields on the structure, PoD and PFA should be indexed by the coordinates $\boldsymbol{x}$ of an inspected point. Here, we consider that the defect is produced by a deterioration mechanism indexed by space $x$ and time $t$ and can be modelled with a space-time stochastic process $d(x, t, \theta)$, where $\theta$ denote the elementary event of an abstract probability space.

\subsection{Definitions of PoD and PFA for stochastic deterioration model}

After inspection with a NDT tool, the measurement of defect $d(\boldsymbol{x}, t, \theta)$ is $\hat{d}(\boldsymbol{x}, t, \theta)$, the 'signal+noise' stochastic field. Then the noise $\eta(\boldsymbol{x}, t, \theta)$ is defined from the knowledge of these two stochastic processes by (4). $\eta(\boldsymbol{x}, t, \theta)=\hat{d}(\boldsymbol{x}, t, \theta)-d(\boldsymbol{x}, t, \theta)$

From Eqs. (2) and (3), PoD and PFA are thus functions indexed by $\boldsymbol{x}$ and $t$ like ROC curves. The process $\hat{d}$ at a given time $t$ being assessed from inspection, the characterization of NDT tools by these curves requires the knowledge of one of the other stochastic processes in Eq. (4): $d$ or $\eta$. Two situations can be considered:

(1) The noise is known because it does not depend on the location of the NDT tool on the structure or because it is known on given areas on the structure. It is generally time invariant and zero mean.

(2) The real size is known because it has been measured before on-site inspections as in ICON project [27] or because an assumption is made.

In both situations the definition of continuous spatial functions needs the complete characterization of the stochastic processes by their marginal distribution and spatial covariance. Practically, almost all NDT tools give data on specific locations and marginal distributions are thus obtained. Moreover, the distance between measurements is generally larger than the distance of correlation and additional assumptions on the structure of correlation for the stochastic processes are needed. Finally note that the knowledge of ageing laws for $d$ allows defining the time dependence of ROC curves. For example for corrosion processes, several models are available [29-31].

\subsection{Statistical approach in the case of repetitive tests with known bias}

Starting from results of a specific NDT testing, we suppose that we get $n_{r}$ repetitive NDT measurements for particular positions $x_{j}$ on the structure and given times $t_{l}$. We denote these measurements by $\left\{\hat{d}_{j, l}^{(i)}\right\}_{i=1}^{n_{r}}$ and consider them as $n_{r}$ outcomes of $\hat{d}\left(x_{j}, t_{l}, \theta\right)$. We consider that an outcome $d_{j . l}$ of the real size $d\left(x_{j}, t_{l}, \theta\right)$ is assessed as follow from these $n_{r}$ repetitive NDT measurements that cover the whole set of noise sources and with bias $b$ :

$d_{j, l}=\frac{1}{n_{r}} \sum_{i=1}^{n_{r}} \hat{d}_{j, l}^{(i)}-b$

If $b$ is a variable (space and time independent) it can be evaluated from a specific NDT testing. If not, expert judgement and inspection process analysis can provide values or bounds for it. Then we deduce $n_{r}$ outcomes $\eta_{j, l}^{(i)}$ of noise $\eta\left(x_{j}, t_{l}, \theta\right)$ as follows:

$\eta_{j, l}^{(i)}=\hat{d}_{j, l}^{(i)}-d_{j, l} \quad$ with $\quad i \in\left\{1, \ldots, n_{r}\right\}$

Of course, a NDT testing on a given structure gives only one outcome of the real size $d$ and $n_{r}$ outcomes of noise $\eta$. In practice, some assumptions on stochastic processes (stationarity, ergodicity, correlation length inferior to the distance between measurements) allow considering measurements at different locations and/or different times as independent outcomes of a random variable. Thus the characterization of marginal distributions of initial stochastic processes can be assessed from the unique available outcome. This will be illustrated in the following sections.

\section{Practical assessment in the case of corrosion of coastal structures in steel}

\subsection{Structures considered and inspection protocol}

We consider here $d$ as the space-time stochastic corrosion process of piles or sheet-piles in coastal regions. The French Center for Maritime and Fluvial Technical Studies (CETMEF) is part of the French ministry of building. It is devoted to the diffusion of 


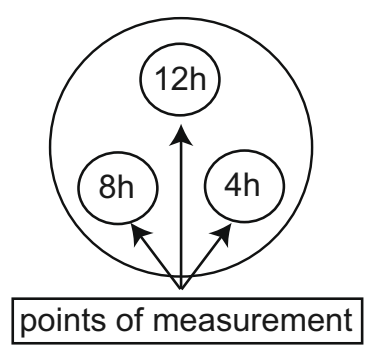

Fig. 5. Location of the three NDT meaurements on a joint.

knowledge, to provide technical and research studies as well as engineering and expert's reports. The CETMEF has edited a protocol for inspection of coastal steel structures through ultrasonic NDT tools. It consists in making ultrasonic measurements of the steel thickness at different levels. The corrosion products are removed by grinding. By using this technique and considering the harsh conditions for marine inspections, the noise on measurements at a given point on the structure cannot be neglected. Thus three inspections are performed on a given point: the three measurements are distributed on a circle, with diameter of about $5 \mathrm{~cm}$, every $120^{\circ}$ as shown on Fig. 5. With these measurements and the initial thickness of the pile that has been reported on the design plans, it is possible to determine the measured loss of thickness $\hat{d}$ mainly due to corrosion and thus the loss of thickness $d$ from Eq. (5).

\subsection{Presentation of the studied structure and data analysis}

The benchmark structure is a wharf located near St. Nazaire city, France (see Fig. 6). Nantes-St Nazaire harbour is the fourth biggest harbour in France. This wharf belongs to the category of on-piles wharves. Building steps and other technical information on these structures can be found in [32]. It is located in the estuary of river Loire near between Nantes and Saint Nazaire towns. It is the second station of a container wharf with 4 stations. Being in the marine environment, the steel piles are susceptible to corrosion and hence the assessment of the structural health of such a structure is considered to be of great practical importance. In fact steel piles play two major roles: the first one is a mechanical function and the second one a protection of reinforced concrete inside against corrosion. The corrosion of these piles is generally uniform corrosion and only this kind of damage is studied in this paper. Eighteen piles have been inspected. At a given height on a pile, the corrosion can be distributed non-uniformly around the pile. It is due to currents, wind and vortex shedding which modify the distribution of dissolved oxygen and nutrients around the pile. Thus the protocol should be completed to assess if this phenome-

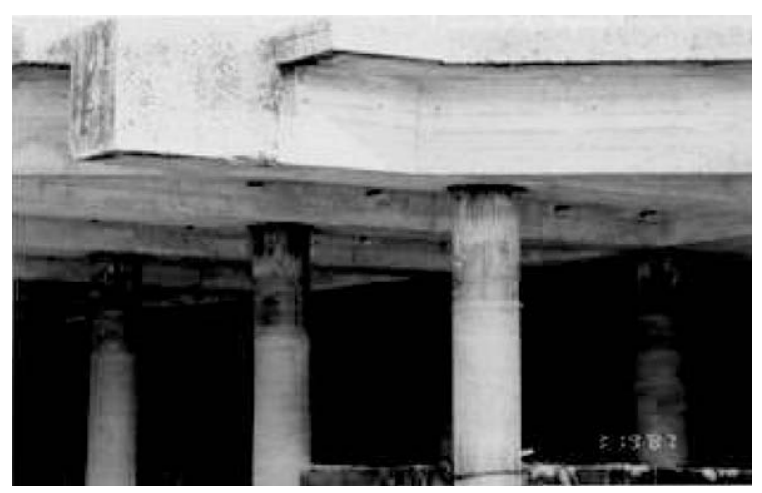

Fig. 6. Steel piles with corrosion.

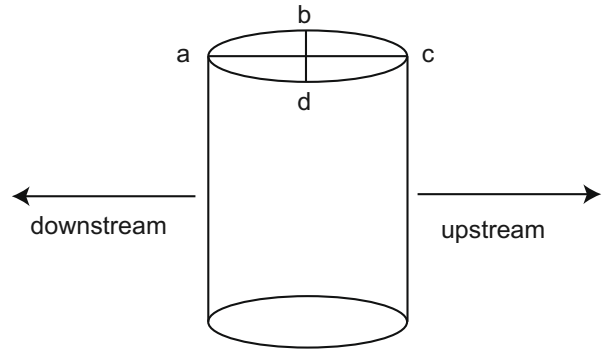

Fig. 7. Cardinal points around the pile at a given height.

non occurs or not. To this aim four areas (cardinal points) are selected around the pile at a given level (see Fig. 7). The vector of spatial indexes $\boldsymbol{x}$ of stochastic processes is the pile number, the vertical abscissa $z$ (reference level: mean sea level) oriented upwards and the cardinal position (North, West, South, East).

For this benchmark structure, no correlation was found between different cardinal positions around the pile for the loss of thickness. Moreover, no correlation was found between the different piles at a given height $z$. Under usual hypotheses on stochastic processes (see Section 3.3), we can then consider the available data (for $d, \hat{d}$ and $\eta$ ) at different cardinal points and for different piles as independent outcomes of stochastic processes indexed by $z$ and having the same probabilistic characterization as marginal stochastic processes. With an abuse of notation, we denote by $\hat{d}(z, \theta), d(z, \theta)$ and $\eta(z, \theta)$ the respective stochastic fields "measured defect", "real defect" and "noise". As three measurements are made on each location of four generatrix and 18 piles ( 72 generatrix in total) at the same height $z_{j}, 216$ outcomes are available for $\hat{d}\left(z_{j}, \theta\right)$ and $\eta\left(z_{j}, \theta\right)$ and 72 outcomes are available for $d\left(z_{j}, \theta\right)$. Here the structure is inspected at six heights: $z_{1}=+2 \mathrm{~m}$ and $z_{2}=+1 \mathrm{~m}$ for tidal zone, $z_{3}=+0.5 \mathrm{~m}, z_{4}=0 \mathrm{~m}, z_{5}=-0.5 \mathrm{~m}$ and $z_{6}=-1 \mathrm{~m}$ for underwater zone.

\subsection{Loss of thickness and noise modeling from assumption on the exact} value

We denote the available outcomes for $\hat{d}\left(z_{j}, \theta\right)$ and $\eta\left(z_{j}, \theta\right)$ by $\hat{d}_{j}^{(i, k)}$ and $\eta_{j}^{(i, k)}$, with $(i, k) \in\left\{1 \ldots n_{r}\right\} \times\left\{1 \ldots n_{p}\right\}$. We denote the outcomes of $d\left(z_{j}, \theta\right)$ by $d_{j}^{(k)}$, with $k \in\left\{1 \ldots n_{p}\right\}$. Here $n_{r}=3$ and $n_{p}=72$. Following Section 3.3, outcomes of $d$ and $\eta$ are deduced from outcomes of $\hat{d}$ as follows:

$d_{j}^{(k)}=\frac{1}{n_{r}} \sum_{i=1}^{n_{r}} \hat{d}_{j}^{(i, k)}-b, \quad$ for $k \in\left\{1 \ldots n_{p}\right\}$

$\eta_{j}^{(i, k)}=\hat{d}_{j}^{(i, k)}-d_{j}^{(k)}, \quad$ for $(i, k) \in\left\{1 \ldots n_{r}\right\} \times\left\{1 \ldots n_{p}\right\}$

From expert judgement (interview of diver and corrosion specialist), the protocol doesn't introduce a systematic bias on the measurement, i.e $b=0$. The corresponding discrete model of the noise (defined at each height) is called model 1.

\subsubsection{Loss of thickness and noise modelling considering a priori classical distributions}

In order to fit the loss of thickness $\hat{d}$ and the noise $\eta$ at a given height $z_{j}$, we use three a priori classical distributions: a Normal distribution, a Generalized Extreme Values distribution (GEV) and a Student distribution. Their probability density functions are given in Table 1. We focus on two heights: $z_{1}=+2 \mathrm{~m}$ and $z_{6}=-1 \mathrm{~m}$ which, respectively belong to tidal and underwater zones. Figs. 8 and 9 show the fittings with these theoretical probability density functions (pdf) and Tables 2 and 3 give the corresponding parameters for each one. By using the minimum of the (-log(likelihood)) 
Table 1

Probability density functions for normal, generalized extreme values and student distributions.

\begin{tabular}{lll}
\hline Fitting & pdf & Parameters \\
\hline Normal & $f(x)=\frac{1}{\sigma \sqrt{2 \pi}} e^{\frac{-(x-\mu)^{2}}{2 \sigma^{2}}}$ & $\mu, \sigma$ \\
GEV & $f(x)=\sigma^{-1} e^{-\left(1+k \frac{(x-\mu)}{\sigma}\right)^{-\frac{1}{k}}}\left(1+k \frac{(x-\mu)}{\sigma}\right)^{-1-\frac{1}{k}}$ & $\mu, \sigma, k$ \\
Student & $f(x)=\frac{\Gamma\left(\frac{v+1}{2}\right)}{\sigma \sqrt{v \pi} \Gamma\left(\frac{v}{2}\right)}\left[\frac{v+\left(\frac{x-\mu}{\sigma}\right)^{2}}{v}\right]^{-\left(\frac{v+1}{2}\right)}$ & $\mu, \sigma, v$ \\
\hline
\end{tabular}

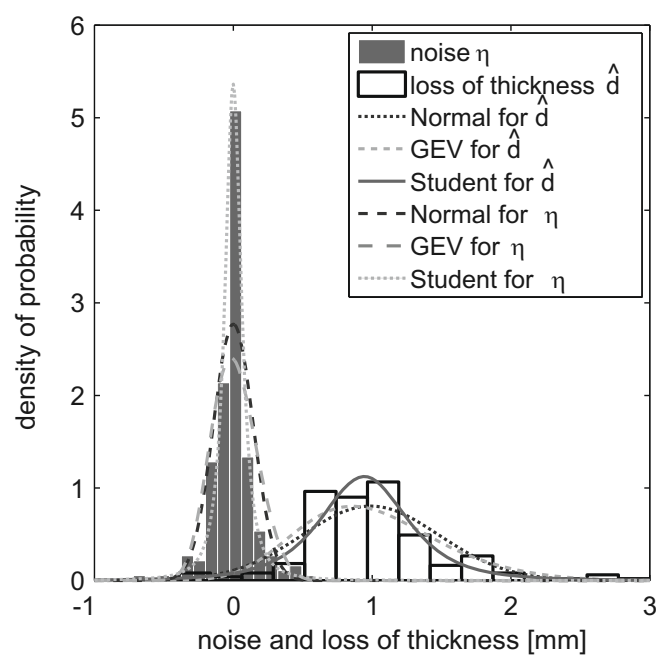

Fig. 8. Noise and loss of thickness fitting with several predefined pdf; $z_{1}=+2 \mathrm{~m}$.

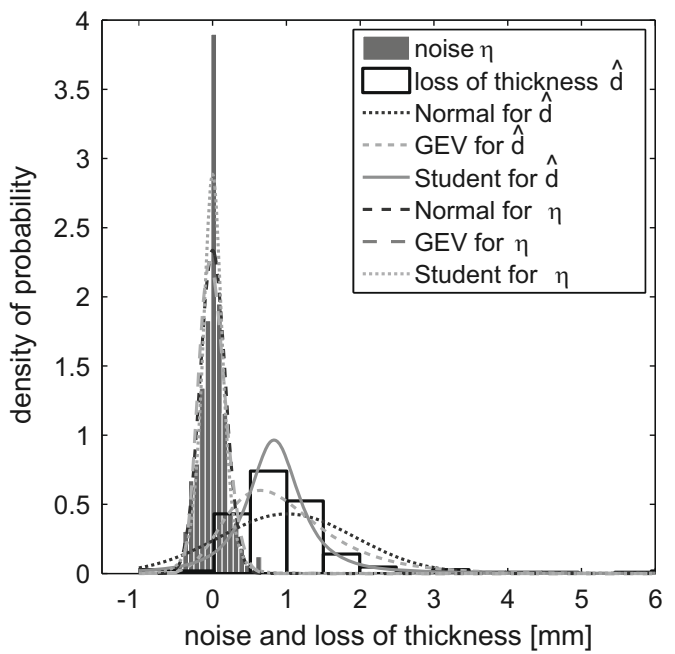

Fig. 9. Noise and loss of thickness fitting with several predefined pdf; $z_{6}=-1 \mathrm{~m}$.

Table 2

Parameters of loss of thickness and noise with classical distributions at $z_{1}=+2 \mathrm{~m}$.

\begin{tabular}{lll}
\hline Fitting & Parameters \\
\cline { 2 - 3 } & Loss of thickness & Noise \\
\hline Normal & $\mu=0.98 ; \sigma=0.49$ & $\mu=2.810^{-4} ; \sigma=0.14$ \\
GEV & $\mu=0.79 ; \sigma=0.46 ; k=-0.14$ & $\mu=-5.3910^{-2} ; \sigma=0.16 ; k=-0.22$ \\
Student & $\mu=0.94 ; \sigma=0.33 ; v=3.12$ & $\mu=-6.310^{-4} ; \sigma=6.510^{-2} ; v=1.74$ \\
\hline
\end{tabular}

Table 3

Parameters of loss of thickness and noise with classical distributions at $z_{6}=-1 \mathrm{~m}$.

\begin{tabular}{lll}
\hline Fitting & Parameters \\
\cline { 2 - 3 } & Loss of thickness & Noise \\
\hline Normal & $\mu=1.01 ; \sigma=0.93$ & $\mu=-1.910^{-4} ; \sigma=0.17$ \\
GEV & $\mu=0.66 ; \sigma=0.61 ; k=0.02$ & $\mu=-6.8410^{-2} ; \sigma=0.17 ; k=-0.17$ \\
Student & $\mu=0.83 ; \sigma=0.37 ; v=1.95$ & $\mu=9.5710^{-5} ; \sigma=0.13 ; v=4.45$
\end{tabular}

Table 4

MLE for several fitting of loss of thickness and noise at $z_{1}=+2 \mathrm{~m}$ and $z_{6}=-1 \mathrm{~m}$.

\begin{tabular}{llllll}
\hline Fitting & $z_{1}=+2 \mathrm{~m}$ & & $z_{6}=-1 \mathrm{~m}$ & \\
\cline { 2 - 3 } \cline { 5 - 6 } & Loss of thickness & Noise & & Loss of thickness & Noise \\
\hline Normal & 154 & -112 & & 289 & -76 \\
GEV & 152 & -100 & & 232 & -73 \\
Student & 139 & -149 & & 209 & -82 \\
\hline
\end{tabular}

estimate (MLE), we see in Table 4 that the Student distribution gives the best fit for both loss of thickness and noise.

\subsubsection{Assessment of ROC points and curves with classical fitting}

The size of samples being large, Eqs. (2) and (3) provide the following rather good approximation of PoD and PFA at each inspected height:

$\operatorname{PoD}\left(z_{j}\right) \approx \frac{\operatorname{Card}\left(A\left(z_{j}\right)\right)}{n_{p} \times n_{r}}, \quad$ with $\quad A\left(z_{j}\right)=\left\{(i, k) \in \mathscr{I} ; \hat{d}_{j}^{(i, k)}>a_{d}\right\}$
$\operatorname{PFA}\left(z_{j}\right) \approx \frac{\operatorname{Card}\left(B\left(z_{j}\right)\right)}{n_{p} \times n_{r}}, \quad$ with $\quad B\left(z_{j}\right)=\left\{(i, k) \in \mathscr{I} ; \eta_{j}^{(i, k)}>a_{d}\right\}$

where $\operatorname{Card}(\cdot)$ indicates the cardinal of a particular set and where $\mathscr{I}=\left\{1 \ldots n_{r}\right\} \times\left\{1 \ldots n_{p}\right\}$. Considering model 1 , these points have been calculated by fixing detection threshold at arbitrary values with a step of $0.05 \mathrm{~mm}$. By linking these points by segments of line, we obtain ROC curves without any fitting: they are denoted by experimental ROC curves in the following. Considering the classical distributions presented in the previous section, we can build the corresponding ROC curves for each selected pdf and compare them to experimental ROC curves coming directly from the experimental data. Considering model 1 for noise, ROC points and curves are plotted on Figs. 10 and 11 for heights $z_{1}=+2 \mathrm{~m}$ and $z_{6}=-1 \mathrm{~m}$. In order to simply quantify the performance of a non-destructive-technique, we can get, from the ROC curve, the distance between the curve and the best performance point with coordinate $[0,1]$ as suggested in [11]: this distance $\delta$ characterizes the optimal efficiency of the NDT tool under specific conditions (detection threshold, conditions of inspection, etc.). Table 5 indicates those distances $\delta$ and the corresponding optimal detection threshold $a_{d}$. According to these figures and table, two remarks are relevant:

- First, we notice that inspections performed at $z_{1}=+2 \mathrm{~m}$ lead to a shorter distance $\delta$ than inspections performed at level $z_{6}=-1 \mathrm{~m}$ : actually, we observe that all inspections performed in the tidal zone are more effective than inspections performed in underwater zone. The diver being the same, the noise is mainly governed by the area to be inspected: in tidal or splash zone, the conditions are good when in underwater zone they are harsh.

- Secondly, about the fitting using predefined distributions laws, it is clear that they don't lead to the best representation especially at $z_{6}=-1 \mathrm{~m}$. Thus, this way of modelling the loss of thickness and noise does not represent well the real performance of the NDT tool and tends to underestimate its efficiency. 


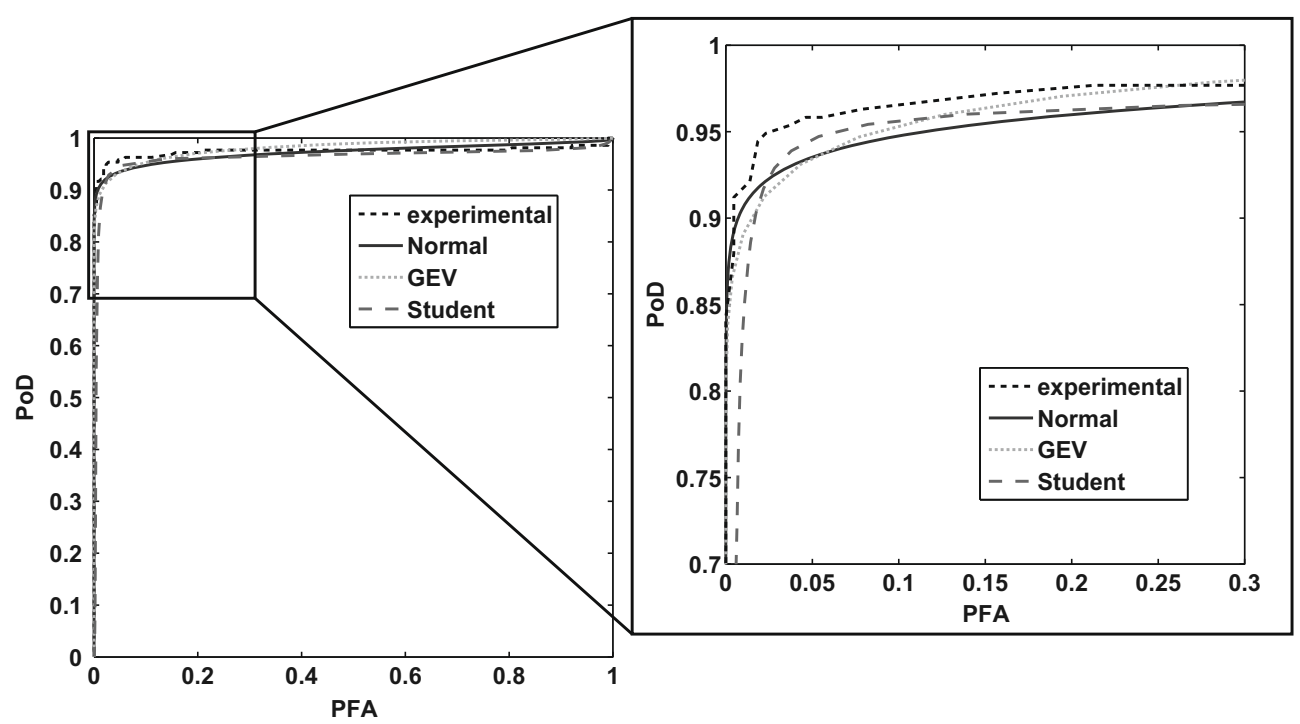

Fig. 10. Comparison between ROC curves coming from predefined distributions and experimental data; $z_{1}=+2 \mathrm{~m}$.

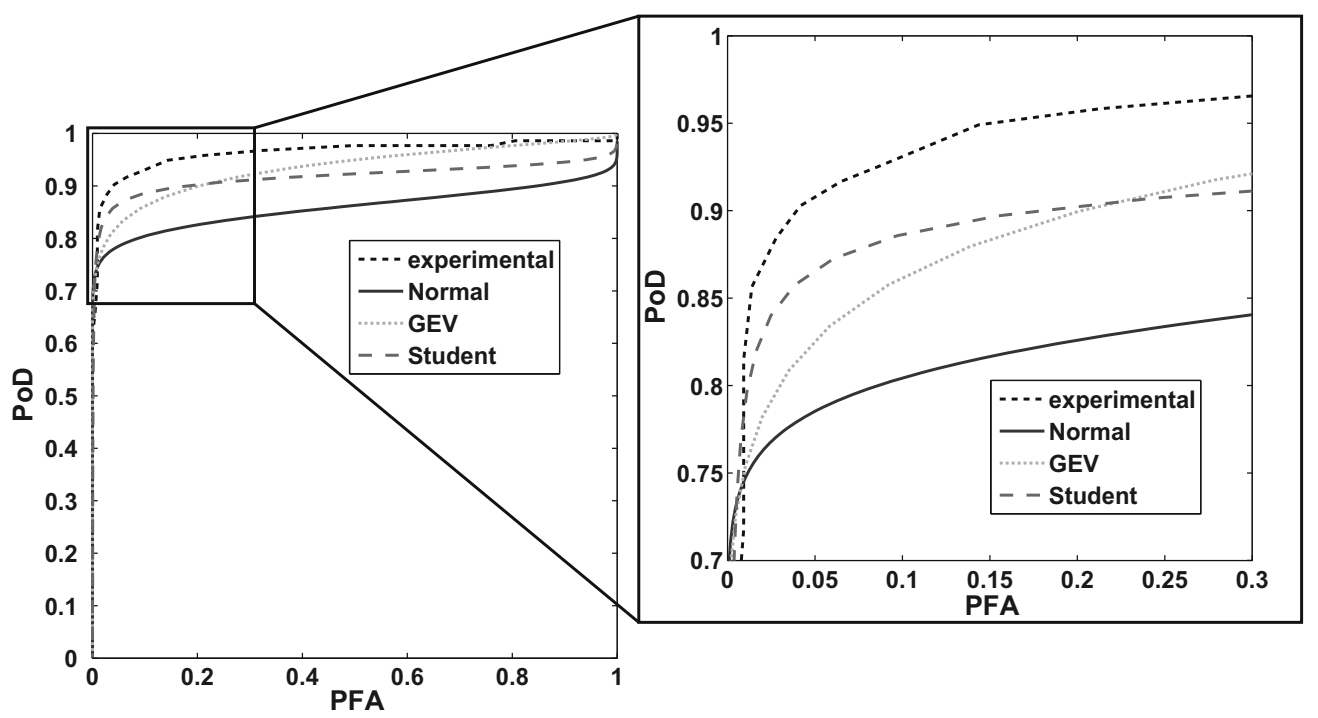

Fig. 11. Comparison between ROC curves coming from predefined distributions and experimental data; $z_{6}=-1 \mathrm{~m}$.

Table 5

Distances $\delta$ and corresponding detection threshold $a_{d}$ for ROC curves coming from classical fittings at level $z_{1}=+2 \mathrm{~m}$ and $z_{6}=-1 \mathrm{~m}$.

\begin{tabular}{llllll}
\hline ROC curves & \multicolumn{2}{l}{$z_{1}=+2 \mathrm{~m}$} & & \multicolumn{2}{l}{$z_{6}=-1 \mathrm{~m}$} \\
\cline { 2 - 3 } \cline { 5 - 6 } & $\delta$ & $a_{d}$ & & $\delta$ & $a_{d}$ \\
\hline Experimental & 0.054 & 0.25 & & 0.105 & 0.25 \\
Normal & 0.079 & 0.26 & 0.211 & 0.25 \\
GEV & 0.081 & 0.29 & 0.169 & 0.25 \\
Student & 0.072 & 0.25 & 0.141 & 0.25
\end{tabular}

From this second remark, the question is: is it possible to get a better fitting of loss of thickness measurements and corresponding noise? Here, we will use the decomposition on polynomial chaos and show that it is a useful tool for this work.

4.3.3. Assessment of ROC curves from decomposition of loss of thickness and noise on polynomial chaos

The method of identification on PC decomposition and the corresponding algorithm is available in $[34,35]$. It lies on the estimate of maximum likelihood [31]. Let $X(\theta)$ be a second order random variable to be identified from $N$ samples, denoted by $\left\{X^{(k)}\right\}_{k=1}^{N}$. An expansion of this random variable on the hermite polynomial chaos writes:

$X(\theta)=\sum_{i=0}^{p} h_{i}(\xi(\theta)) X_{i}$

where $\xi$ is a standard gaussian random variable, $h_{i}$ is the normalized Hermite polynomial of degree $i$ and $p$ is the order of the polynomial chaos expansion. The aim of the identification procedure is to find the coefficients $X_{i}$ of the decomposition. We here assume that mean and standard deviation are rather well estimated from samples. Due to orthonormality properties of Hermite polynomials, it gives the following constraints on the coefficients: denoting by $\mu_{\exp }$ and $\sigma_{\exp }$ the mean and standard deviation obtained from samples, mean $\mu_{X}$ and standard deviation $\sigma_{X}$ of $X(\theta)$ are rather well estimated from $\mu_{\text {exp }}$ and $\sigma_{\text {exp }}$ and constraint the decomposition to satisfy the following conditions on the coefficients: 
$-\log \left(\mathrm{L}\left(\phi_{1}, \phi_{2}\right)\right)$
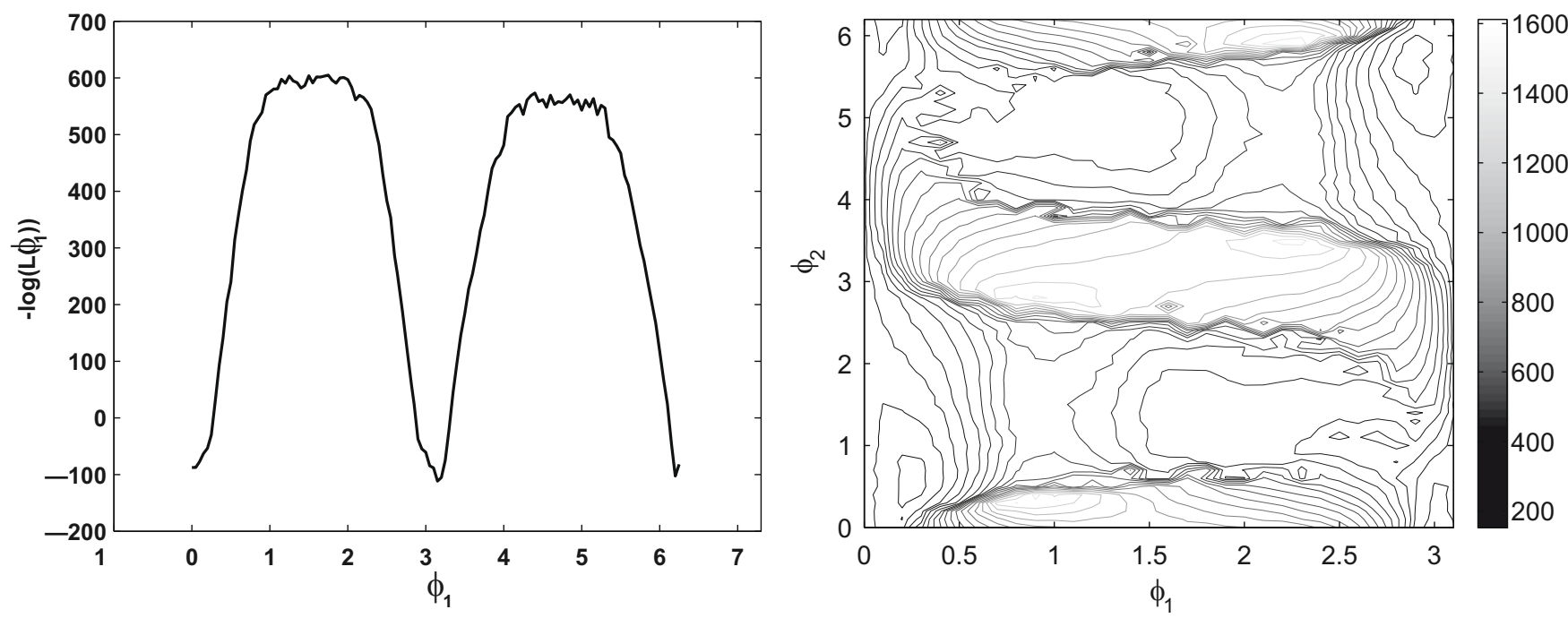

Fig. 12. Evolution of $-\log (L(\phi b))$ for PC order $p=2$ and $p=3$; noise at $z_{1}=+2 \mathrm{~m}$.

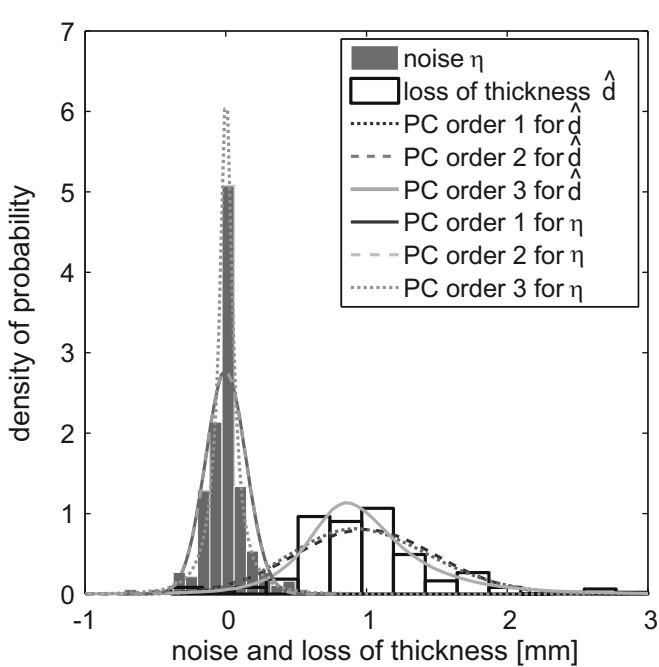

Fig. 13. Noise and of loss of thickness fitting with polynomial chaos for several orders $p ; z_{1}=+2 \mathrm{~m}$.

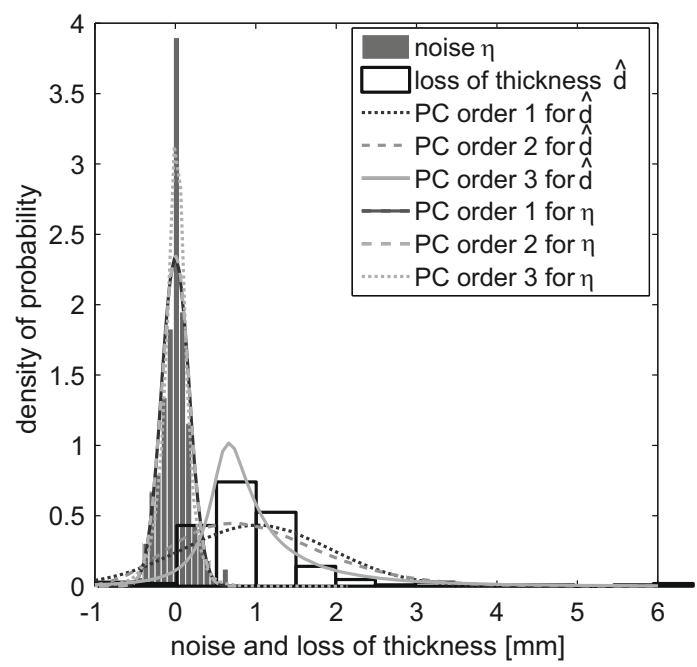

Fig. 14. Noise and of loss of thickness fitting with polynomial chaos for several orders $p ; z_{6}=-1 \mathrm{~m}$.

$$
\begin{aligned}
& X_{0}=\mu_{\text {exp }} \\
& \sum_{i=1}^{p} X_{i}^{2}=\sigma_{\text {exp }}^{2}
\end{aligned}
$$

Letting $a_{i}=X_{i} / \sigma_{X}$, the PC decomposition can be rewritten as follows:

$X(\theta)=\mu_{\exp }+\sigma_{\exp } \sum_{i=1}^{p} a_{i} h_{i}(\xi(\theta))$

where coefficients $a_{i}$ must satisfy:

$\sum_{i=1}^{p} a_{i}^{2}=1$

Denoting by $\mathbf{a}=\left(a_{1}, \ldots, a_{p}\right)^{T} \in \mathbb{R}^{p}$, we denote by $p_{X}(\cdot ; \mathbf{a})$ the probability density function of $X$, parametrized by a, and introduce the likelihood function:

$L(\mathbf{a})=\prod_{k=1}^{N} p_{X}\left(X^{(k)} ; \mathbf{a}\right)$

Table 6

MLE for several PC fitting of loss of thickness and noise at $z_{1}=+2 \mathrm{~m}$ and $z_{6}=-1 \mathrm{~m}$.

\begin{tabular}{llllll}
\hline Fitting & $z_{1}=+2 \mathrm{~m}$ & & & $z_{6}=-1 \mathrm{~m}$ \\
\cline { 2 - 3 } \cline { 6 - 6 } & Loss of thickness & Noise & & Loss of thickness & Noise \\
\hline PC order 1 & 154 & -98 & & 326 & -76 \\
PC order 2 & 150 & -109 & & 256 & -76 \\
PC order 3 & 132 & -155 & & 202 & -85 \\
\hline
\end{tabular}

Table 7

Distances $\delta$ and corresponding detection threshold $a_{d}$ for ROC curves coming from PC fittings at level $z_{1}=+2 \mathrm{~m}$ and $z_{6}=-1 \mathrm{~m}$

\begin{tabular}{llllll}
\hline ROC curves & \multicolumn{2}{l}{$z_{1}=+2 \mathrm{~m}$} & & \multicolumn{2}{l}{$z_{6}=-1 \mathrm{~m}$} \\
\cline { 2 - 3 } \cline { 5 - 6 } & $\delta$ & $a_{d}$ & & $\delta$ & $a_{d}$ \\
\hline Experimental & 0.054 & 0.25 & & 0.105 & 0.25 \\
PC order 1 & 0.079 & 0.26 & & 0.22 & 0.23 \\
PC order 2 & 0.072 & 0.26 & 0.22 & 0.23 \\
PC order 3 & 0.052 & 0.26 & & 0.102 & 0.25
\end{tabular}




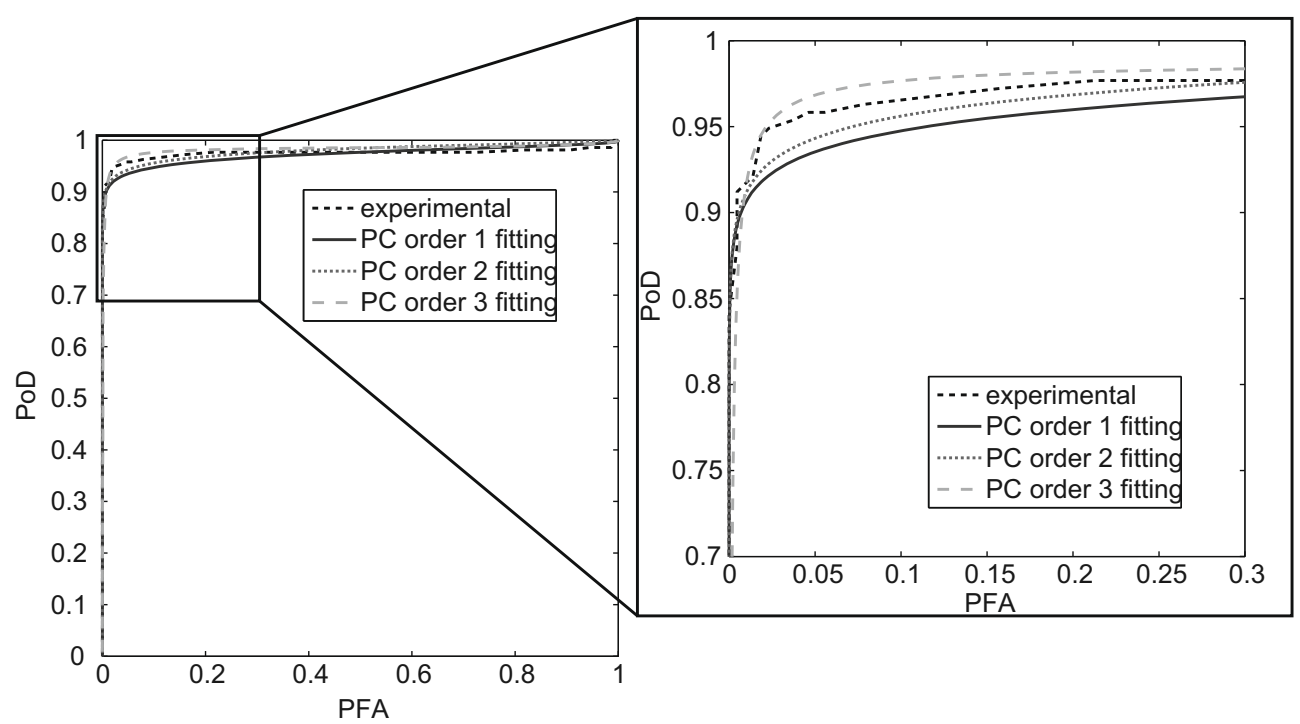

Fig. 15. Comparison between ROC curves coming from polynomial chaos identification and experimental data; $z_{1}=+2 \mathrm{~m}$.

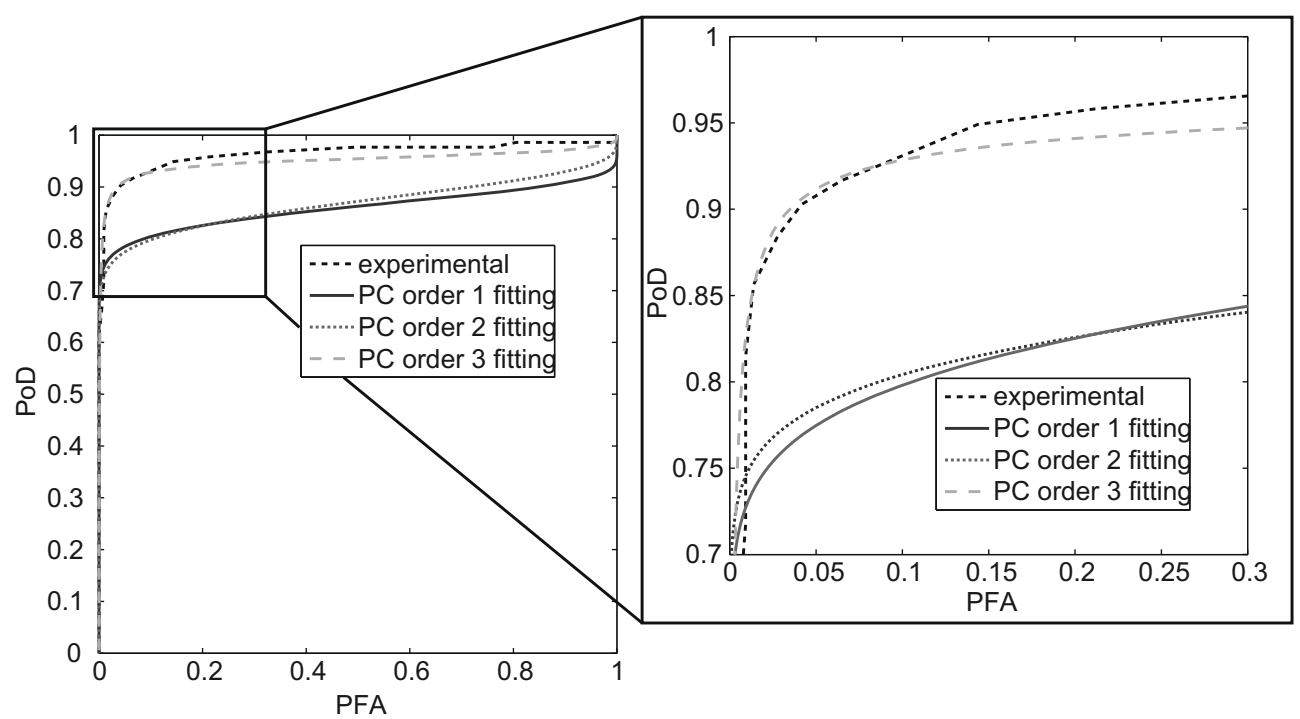

Fig. 16. Comparison between ROC curves coming from polynomial chaos identification and experimental data; $z_{6}=-1 \mathrm{~m}$.
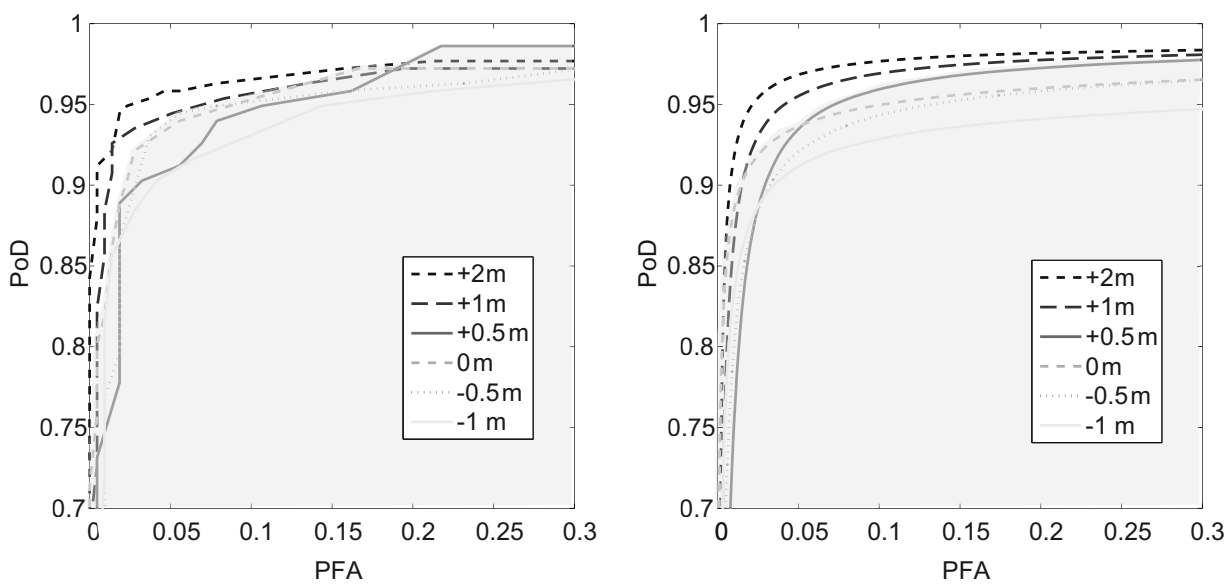

Fig. 17. Comparison between ROC curves coming from polynomial chaos with order 3 identification (left) and experimental data (right) for all levels 


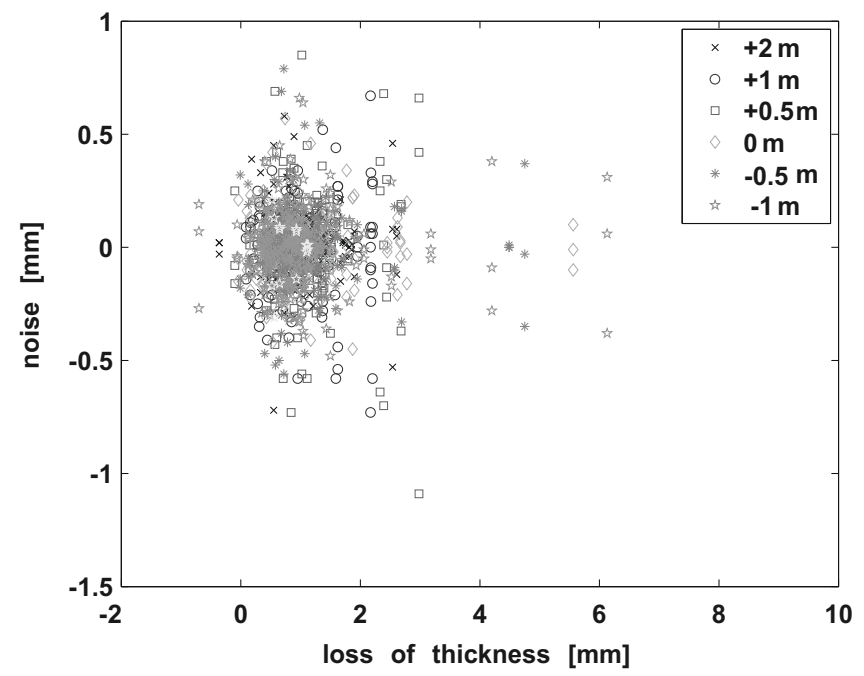

Fig. 18. Scatter of points $[\bar{d} ; \eta]$ for all levels (216 points for each level).

Maximum likelihood estimation is a popular statistical method used for fitting a mathematical model to a database. Here, it is used to fit a pdf. The aim is then to find a that maximizes $L(\mathbf{a})$. The identification problem then writes: find a such that

$$
\begin{gathered}
-\log (L(\mathbf{a}))=\operatorname{argmin} \\
\mathbf{a} \in \mathbb{R}^{p} \quad(-\log (L(\mathbf{a}))) \\
\mathbf{a}^{T} \mathbf{a}=1
\end{gathered}
$$

This is an optimization on the unit hypersphere of $\mathbb{R}^{p}$. In practice, we adopt the following characterization of the hypersphere:

$$
\left\{\begin{array}{l}
a_{1}=\cos \left(\phi_{1}\right) \\
a_{i}=\cos \left(\phi_{n}\right) \prod_{i=1}^{n-1} \sin \left(\phi_{i}\right) \quad \text { for } \quad i \in\{2, \ldots, p-1\} \\
a_{p}=\prod_{i=1}^{p-1} \sin \left(\phi_{i}\right)
\end{array}\right.
$$

with $\phi_{p-1} \in[0,2 \pi]$ and $\forall i \in\{1, \ldots p-1\}, \phi_{i} \in[0, \pi]$. The optimization problem is then reformulated as an unconstrained optimization problem on $\mathbb{R}^{p-1}$. It is solved by a two step procedure:

(1) a coarse localization of a minimum is found through a basic random search algorithm;

(2) starting from this point, the Nelder-Mead Simplex Method is used [36].

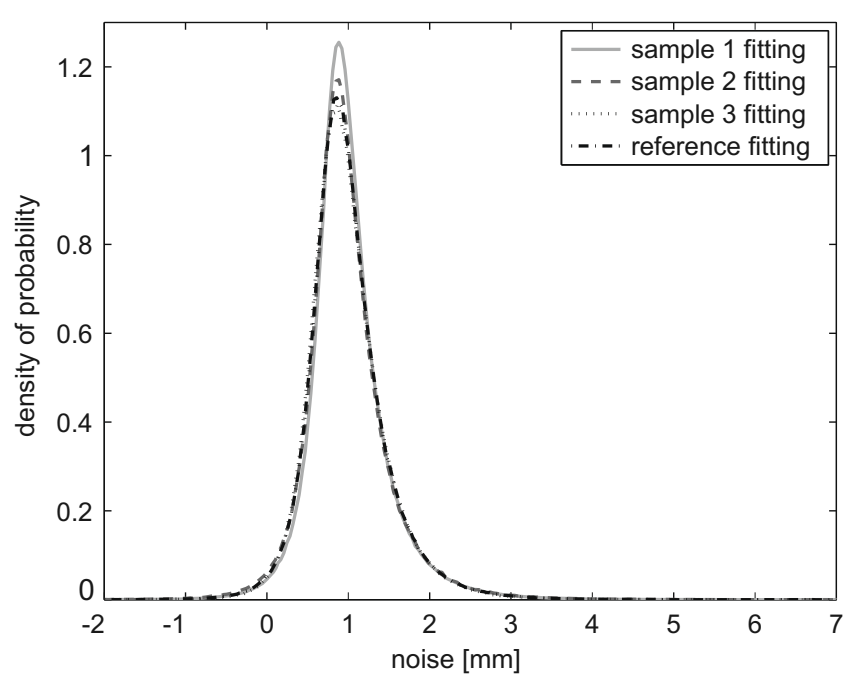

Fig. 20. Loss of thickness modelling using model 2 as random noise generator; $z_{1}=+2 \mathrm{~m}$.

This problem has generally several local minima (see Fig 12), and it is convenient to repeat this two-step procedure in order to find the global solution (for instance, 10 times for a PC of degree $p=2$, and 100 times for a PC of degree $p=3$ ).

For our application, this identification on polynomial chaos is performed separately on the measured loss of thickness $\hat{d}$ and the noise $\eta$ at each height. Figs. 13 and 14 present the fitting results with the method of identification on PC decomposition for order $p \in\{1,2,3\}$, respectively at $z_{1}$ and $z_{6}$. Table 6 indicates the MLE for loss of thickness and noise: clearly, with a PC order $p=3$ we get better fittings than with classical distributions. (Table 7)

At a given height, the aim is to get the ROC curves, i.e. PoD and PFA, using polynomial chaos decomposition of loss of thickness $\hat{d}$ and noise $\eta$. Denoting by $\left\{\hat{d}_{j, i}\right\}_{i=0}^{p}$ and $\left\{\eta_{j, i}\right\}_{i=0}^{p}$ the coefficients of the PC decomposition of $\hat{d}\left(z_{j}, \theta\right)$ and $\eta\left(z_{j}, \theta\right)$, we obtain the following approximation of PoD and PFA:

$P o D=\sum_{i=0}^{p} \hat{d}_{j, i} \int_{a_{d}}^{\infty} h_{i}(u) p_{\xi}(u) d u$
$P F A=\sum_{i=0}^{p} \eta_{j, i} \int_{a_{d}}^{\infty} h_{i}(u) p_{\xi}(u) d u$
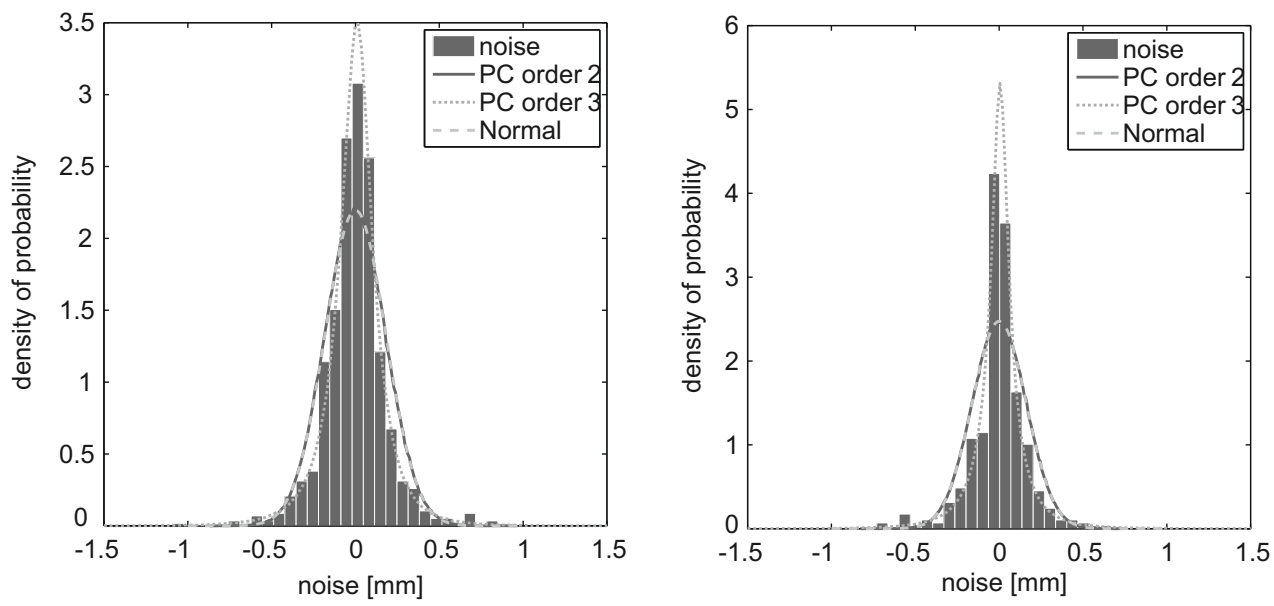

Fig. 19. Histogram of noise and fittings in tidal zone (left) and underwater zone (right). 


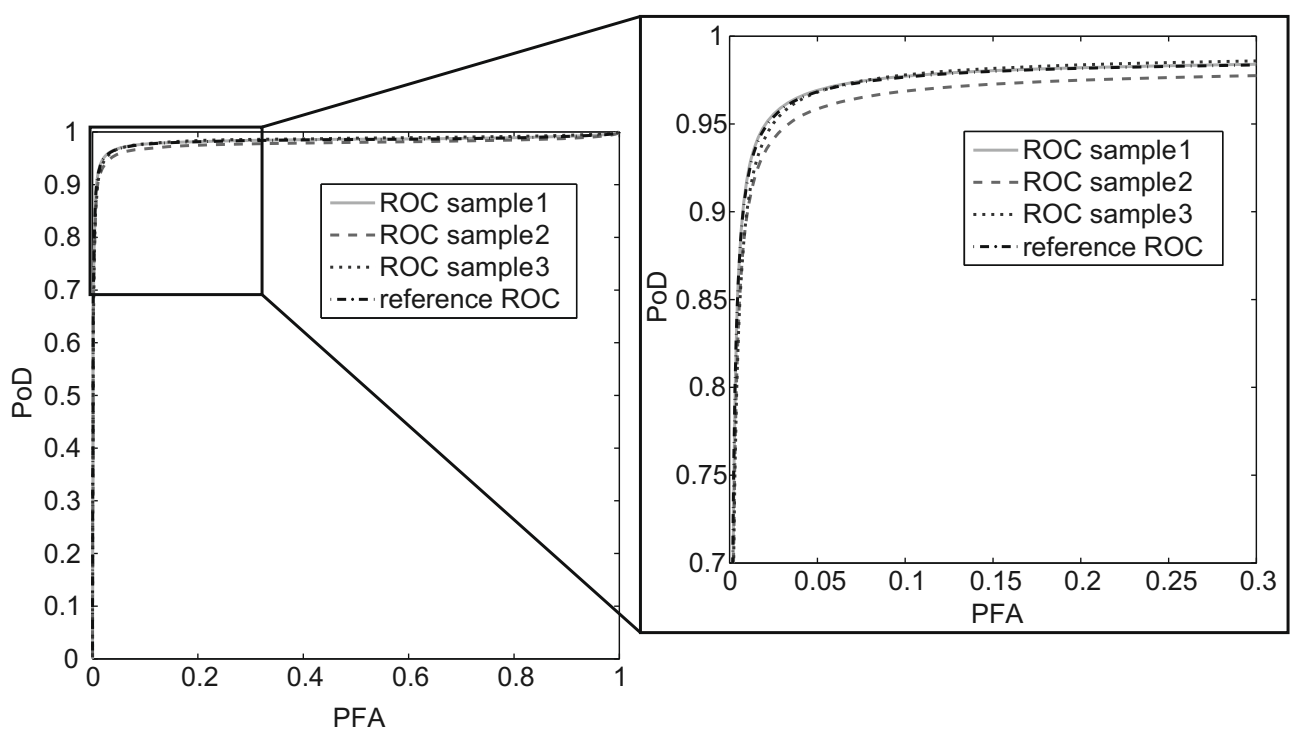

Fig. 21. ROC curves using model 2 as random noise generator; $z_{6}=+2 \mathrm{~m}$.

where $p_{\xi}$ is the measure of probability associated to the standard gaussian random variable $\xi$. Practically, integrals in Eqs. (19) and (20) are computed through Monte-Carlo simulations using $10^{6}$ samples. These quantities are independent of the study: they can be pre-processed once for all and used for each application. As shown in Figs. 15 and 16, the ROC curves coming from polynomial chaos identification with $p=3$ lead to a good estimation of $\delta$ according to experimental ROC curves.

Fig. 17 presents all experimental ROC curves at the 6 levels and the corresponding ROC curves with identification of loss of thickness and noise with PC decomposition. Two families of curves appear. The first one is close to the best ROC point with coordinates $[0,1]$ and the second one is composed of less effective inspection. Note that this second family, in the colored area, gathers all the inspections performed in the underwater zone for which inspection conditions are harsh.

\subsection{Use of another noise modelling}

In this section, we present another model for the noise $\eta$ called model 2. The building of sample of noise (see Eq. (6)) allows plotting the scatter diagram of Fig. 18: it is clearly shown that there is no correlation between the noise and the real size of loss of thickness [33]. Thus we suppose that the noise is mainly governed by the inspected area (tidal or underwater zones). Then, we consider that the noise is a piecewise homogeneous stochastic field depending on the location in the tidal or underwater zone. We also consider that samples at different heights in a given zone are different independent outcomes of a random variable allowing characterizing the marginal distribution of noise in this zone. Distributions of noise in tidal and underwater zones are presented on Fig. 19. A total of 432 measurements were taken in the tidal zone and 864 in the underwater zone. We note that decompositions on a polynomial chaos with order $p=3$ lead to best fittings for the two areas. Considering the real defect size at height $z_{1}=+2 \mathrm{~m}$ and using the previous decomposition on the polynomial chaos with order $p=3$ of the noise for tidal zone, we generate three random samples of loss of thickness. Fig. 20 shows the identifications on the polynomial chaos of these three samples and compare them to the initial identification of the the real loss of thickness at $z_{1}=+2 \mathrm{~m}$. We observe that these new fittings are very close to the initial one: this is showing that model 2 seems accept- able for generating random noise. Finally, Fig. 21 presents ROC curves coming from identifications of these random samples and noise model 2: except for sample 2, we observe that each new ROC curves is very close to the initial one: the reference ROC curve leads to $\delta=0.052$ while ROC curves coming from the random samples, respectively give $\delta_{1}=0.052, \delta_{2}=0.060$ and $\delta_{3}=0.053$. As the experimental ROC curves gives $\delta_{\text {exp }}=0.054$, these results seem good with a maximum error inferior to $10 \%$. For ROC curve calculated with sample 2 the gap is coming from the identification: we have noticed that the distribution's tails were different from the others. This leads to ask if the criteria based on the estimate of maximum likelihood for the identification is a good choice for well fitting the distribution's tails.

\section{Conclusion}

Concepts of PoD, PFA and ROC curves coming from detection theory are very useful tools in order to quantify the quality of non-destructive-techniques. Classically used for inspection of cracks of offshore structures, they can also be applied to corrosion problem in the case of inspection of ships or corroded marine and coastal structures. ROC curves can be easily built in the discrete case but their use in a RBI analysis involves getting them with a continuous formulation of loss of thickness and noise. In this paper fittings of loss of thickness and noise with predefined probability density functions have been performed but we have observed that classical distributions don't lead to correct fitting of data. It is shown that the method of identification based on polynomial chaos leads to better results for order of chaos $p=3$ and allows to obtain rather precise ROC curves according to experimental ROC points. This decomposition is also tractable for Stochastic Finite Element Method [37]. Moreover, two models of noise have been proposed in this paper which have both lead to good results according to experimental ROC curves. Thus, using these noise models would be possible to carry out a RBI analysis.

\section{Acknowledgements}

This work is supported by the European Community and FEDER funds within the MEDACHS Interreg IIIB (Atlantic space, project N197) (MEDACHS: Marine Environment Damage to Atlantic Coast Historical and transport works or Structures: Methods of diagnosis, 
repair and of maintenance) (web site: http://www.medachs.u-bordeaux1.fr). The authors would like to thank Harbour Authorities of Nantes St Nazaire, for their technical support and expert judgement.

\section{References}

[1] Kong JS, Frangopol DM. Life-cycle reliability-based maintenance cost optimization of deteriorating structures with emphasis on bridges. Journal of Structural Engineering ASCE 2003. June.

[2] Kong JS, Frangopol DM. Cost-reliability interaction in life-cycle cost optimization of deteriorating structures. Journal of Structural Engineering ASCE 2004. November.

[3] Kong JS, Frangopol DM. Probabilistic optimization of ageing structures considering maintenance and failure costs. Journal of Structural Engineering ASCE 2005. April.

[4] Lauridsen J, Bjerrum J, Sloth M, Jensen FM. Principles for a guideline for probability-based management of deteriorated bridges. In: IABMAS 2006 conference, Bridge Maintenance, Safety and Management, Porto.

[5] Radojicic A, Bailey SF, Brühwiler E. Probabilistic models of cost for the management of existing structures. Life-Cycle Cost Analysis and Design of Civil Infrastructure Systems, SEI-ASCE 2001. August.

[6] Stewart MG. Reliability based assessment of ageing bridges using risk ranking and life cycle cost decision analyses. Reliability Engineering and System Safety 2001;74:263-73.

[7] Stewart MG, Estes AC, Frangopol DM. Bridge deck replacement for minimum expected cost under multiple reliability constraints. Journal of Structural Engineering, ASCE 2004. September.

[8] Estes AC, Frangopol DM. Repair optimization of highway bridges using system reliability approach. Journal of Structural Engineering, ASCE 1999. July.

[9] Stewart MG, Mullard JA. Life-cycle cost analysis considering spatial and temporal variability of corrosion-induced damage and repair of concrete surfaces. ICOSSAR 2005.

[10] Stewart MG, Mullard JA. Reliability based assessment of the influence of concrete durability on the timing of repair for RC bridges. In: IABMAS 2006 conference, Bridge Maintenance, Safety and Management, Porto.

[11] Schoefs F, Clément A. Multiple inspection modeling for decision making and management of jacket off-shore platforms: effect of false alarms. In: First international forum on engineering decision making (IFED'04). Switzerland: Stoos; December 5-9 2004.

[12] Straub D, Faber MH. Modeling dependency in inspection performance. In: ICASP 2003, Applications of Statistics and Probability in Civil Engineering. Rotterdam; 2003.

[13] Rouhan A, Schoefs F. Probabilistic modeling of inspection results for offshore structures. Journal of Structural Safety 2003;25:379-99.

[14] Faber MH, Sorensen JD. Indicators for inspection and maintenance planning of concrete structures. Journal of Structural Safety 2002;24

[15] Faber MH. RBI: An Introduction. Structural Engineering International 2002;3:187-94.

[16] Onoufriou T, Frangopol DM. Reliability-based inspection optimisation of complex structures: a brief retrospective. Journal of Computers and Structures $2002 \cdot 80$

[17] Goyet J, Paygnard JC, Maroini A, Faber MH. Optimal inspection and repair planning: case studies using IMREL software. In: Proceeding of the 13th international conference on offshore mechanics and arctic engineering, vol. 2. NY: ASME.

[18] Breysse D, Elachaci SM, Sheils E, Schoefs F, O'Connor A. Life cycle cost analysis of ageing structural components based on non destructive condition assessment. Australian Journal of Structural Engineering 2009;9(1):55-66 [special issue: Disaster \& Hazard Mitigation], ISSN: 1328-7982.
[19] Breysse D, Vasconcelos E, Schoefs F. Management Strategies and Improvement of Performances of Sewer Networks. Computer Aided Civil And Infrastructure Engineering 2007;22(7):462-77.

[20] Schoefs F, Rguig M, Risk based inspection of jackets submitted to through-wall cracks. In: Proceeding of 22th international conference on offshore mechanics and arctic engineering (O.M.A.E'03), Proceedings on CD. Cancun, Mexico: ASME; 8-13 June 2003. p. 7.

[21] Arques PY. Decisions en traitement du signal. Paris: Masson; 1982.

[22] Füscök F, Muller C, Scharmach M. Human factors: the NDE reliability of routine radiographic film evaluation. In: Proceedings of the 15 th world conference on non destructive testing, vol. 3; 2000.

[23] Barnouin B, Lemoine L, Dover WD, Rudlin J, Fabbri S, Rebourcet G. Underwater inspection reliability trials for offshore structures. In: Topp D, Kare R, Sangouard D, editors. Proceeding of the 12th international conference on offshore mechanics and Arctic engineering, vol. 3B. NY: ASME; 1993. p. 88390.

[24] Pakrashi V, Schoefs F, Memet JB, OĆonnor A, An image analysis based damage classification methodology. In: Taylor and Francis, editors. Structure and infrastructure engineering: maintenance, management, life-cycle design and performance (NSIE), 2008; doi:10.1080/15732470701718072.

[25] Silk MG. Estimates of the probability of detection of flaws in TOFD data with varying levels of noise. Insight 1996;38(1 January):31-6.

[26] Rudlin JR, Muhammed A, Schneider C. Inspection reliability and periodicity for rail axle inspection. Insight 2006;48(6 June):151-348.

[27] Rudlin JR, Dover WD. The ICON database, Assisting underwater inspections. In: Offshore technology, vol. 4; 1996.

[28] Rudlin JR. Reliability of inspection for fatigue cracks in offshore structures. In: Colloque lEE; 1996. p. 6/1-6/3.

[29] Melchers RE. A new model for marine immersion corrosion in structural reliability assessment. Applications of Statistics and Probability in Civil Engineering 2003.

[30] Paik JK, Lee JM, Park Y, Hwang JS, Kim CW. Time-variant ultimate longitudinal strength of corroded bulk carriers. Marine Structures 2003;16:567-600.

[31] Guedes Soares C, Garbatov Y. Reliability of maintained corrosion protected plates subjected to non-linear corrosion and compressive loads. Marine Structures 1999;12:425-45.

[32] Yánez-Godoy H, Schoefs F, Casari P. Statistical analysis of the effects of building conditions on the initial loadings of on-piles quays, vol. 7, no. 3 . Structural Health Monitoring (SAGE); 2008. p. 245-63. doi: dx.doi.org/ 10.1177/1475921708090563 [published on line April 1st 2008; Published September 1st 2008].

[33] Schoefs F, Clément A, Memet JB, Nouy A. Spatial dependence of receiver operating characteristic curves for risk based inspection of corroded structures: application to on-pile wharf. In: Jun Kanda, Tsuyoshi Takada, Hitoshi Furuta, editors. Proceeding of 10th international conference on applications of statistics and probability in civil engineering (I.C.A.S.P'07). Tokyo, Japan; July 31-August 3 2007. 8 pages.

[34] Schoefs F, Yán̈ez-Godoy H, Nouy A. Identification of random material properties from monitoring of structures using stochastic chaos. In: Jun Kanda, Tsuyoshi Takada, Hitoshi Furuta, editors. Proceeding of 10th international conference on applications of statistics and probability in civil engineering (I.C.A.S.P’07)Tokyo, Japan; July 31-August 3 2007. 8 pages.

[35] Desceliers C, Soize C, Ghanem R. Identification of chaos representations of elastic properties of random media using experimental vibration tests. Computational Mechanics 2007;39:831-8.

[36] Lagarias JC, Reeds JA, Wright MH, Wright PE. Convergence properties of the Nelder-Mead Simplex Method in low dimensions. SIAM Journal of Optimization 1998;9:112-47.

[37] Schoefs F. Risk analysis of structures in presence of stochastic fields of deterioration: coupling of inspection and structural relability. Australian Journal of Structural Engineering 2007;9(1):67-78. ISSN: 1328-7982. 